ISSN 0258-7122 (Print), 2408-8293 (Online)

Bangladesh J. Agril. Res. 43(1): 125-134, March 2018

\title{
COMBINING ABILITY AND HETEROSIS STUDY IN MAIZE (Zea mays L.) HYBRIDS AT DIFFERENT ENVIRONMENTS IN BANGLADESH
}

\author{
A. N. M. S. KARIM ${ }^{1}$, S. AHMED ${ }^{2}$, A. H. AKHI ${ }^{3}$ \\ M. Z. A. TALUKDER ${ }^{4}$ AND T. A. MUJAHIDI ${ }^{5}$
}

\begin{abstract}
The aim of this study was to isolate superior inbred lines and better combining parents for suitable hybrids and to determine percent of heterosis using standard commercial checks in a $7 \times 7$ diallel analysis excluding reciprocals over five environments. The mean sum of square obtained from combined analysis of variance showed the presence of genetic variability among the crosses, environment and crosses $\times$ environment interaction for all of the characters under study. The variances for general combining ability (GCA) and specific combining ability (SCA) of variance were found significant for all the characters. However, relative magnitude of variances indicated that additive gene effects were more prominent for all the characters studied. GCA and SCA effects both showed significant interaction with environment for all the traits. This clearly suggested the need of selecting different parental lines for hybrids for different ecological situations. Parents $\mathrm{P}_{3}, \mathrm{P}_{5}$ and $\mathrm{P}_{6}$ were the best general combiner for high yield; parents $\mathrm{P}_{6}$ for earliness; and $\mathrm{P}_{1}, \mathrm{P}_{2}$ and $\mathrm{P}_{3}$ for dwarf plant type. The range of heterosis expressed by different crosses was from 13.04 to $5.25 \%$ percent for grain yield. The better performing six crosses $\left(\mathrm{P}_{1} \times\right.$ $\mathrm{P}_{2}, \mathrm{P}_{1} \times \mathrm{P}_{5}, \mathrm{P}_{3} \times \mathrm{P}_{4}, \mathrm{P}_{3} \times \mathrm{P}_{6}, \mathrm{P}_{3} \times \mathrm{P}_{7}$ and $\mathrm{P}_{4} \times \mathrm{P}_{5}$ ) can be utilized for developing high yielding hybrid varieties as well as for exploiting hybrid vigour. These crosses also need to be evaluated further in multilocations.
\end{abstract}

Keywords: Combining ability, across locations, maize hybrids.

\section{Introduction}

Maize is one of the oldest and most important crop in the world. It is the highest yielding grain crop having multiple uses. Now maize has become an important cereal crop in Bangladesh. In Bangladesh it is the third most important crop after rice and wheat and it accounts for $4.8 \%$ of the total cropped land area and $3.5 \%$ of the value of agricultural output (Ahmad et al., 2011). In 2015-16, maize was cultivated in 3.5 lakh hectare of land in Bangladesh and production was 25 lakh mtons (Bidan, 2016).

Hybrid maize has much higher yield potentiality than those of synthetics and composites. The hybrid seeds currently being used are imported and involved huge amount of foreign currency. In the recent past, exploitation of hybrid vigour

\footnotetext{
${ }^{1,3 \& 5}$ Scientific Officer, Plant Breeding Division, Bangladesh Agricultural Research Institute (BARI), Gazipur, ${ }^{2}$ Principal Scientific Officer, Plant Breeding Division, BARI, Gazipur, ${ }^{4}$ Senior Scientific Officer, Plant Breeding Division, BARI, Gazipur, Bangladesh.
} 
and selection of parents based on combining ability has been used as an important breeding approach in crop improvement. Developing of high yielding $\mathrm{F}_{1}$ hybrids along with other favorable traits are receiving considerable attention. For developing good hybrids, information about combining ability of the parents and the resulting crosses is essential. The present study involving a $7 \times 7$ diallel analysis over five environments aimed to determine the better general combining parents and for isolating good cross combinations in maize for evolving suitable hybrid(s) locally.

\section{Materials and Methods}

Seven inbred lines of maize viz. $\mathrm{P}_{1}$ (CML429), $\mathrm{P}_{2}$ (CLG1837), $\mathrm{P}_{3}$ (CML285), $\mathrm{P}_{4}$ (CML451), $\mathrm{P}_{5}$ (CML431), $\mathrm{P}_{6}$ (CML223) and $\mathrm{P}_{7}$ (CML551) collected from CIMMYT- Mexico, were crossed in all possible combinations (excluding reciprocals) during rabi season of 2014-2015 at Gazipur. During rabi 2015-2016, the $21 \mathrm{~F}_{1}$ 's and along with three commercial hybrids viz. BHM9, 981 and NK40 were grown following Alpha lattice design with three replications in five different environments viz. Gazipur, Burirhat, Hathazari, Rahmatpur and Jessore. In Gazipur sowing was done on 22 November, 21 November in Burirht, where as it was 30 November in Hathazari, 22 November in Rahmatpur and 1 December in Jessore. Each plot comprised of two rows $4 \mathrm{~m}$ long. Spacing was maintained $0.6 \mathrm{~m}$ between rows and $0.25 \mathrm{~m}$ spacing between plants. One healthy seedling per hill was kept after proper thinning. Fertilizers were applied @ 250, 55, 110, 40,5 and $1.5 \mathrm{~kg} / \mathrm{ha}$ of $\mathrm{N}, \mathrm{P}, \mathrm{K}, \mathrm{S}, \mathrm{Zn}, \mathrm{B}$, respectively. Standard agronomic practices were followed and plant protection measures were taken when required. Data on days to $50 \%$ pollen shedding and silking were recorded on whole plot basis. Ten randomly selected plants were used for recording observations on plant height and ear height. All the plants in two rows were considered for plot yield which was later converted to t/ha. The combining ability analysis was carried out following Model I (fixed effects) and Method IV (one set of $F_{1}$ 's but neither parents nor reciprocal $F_{1}$ 's is included) described by Griffing (1956) in multi environment using PB Tools.

\section{Results and Discussion}

The mean sum of square obtained from combined analysis of variance showed the presence of genetic variability among the crosses and environment for all the characters under studied (Table 1).This indicated influence of differential environmental factors at different locations on the expression of different characters on maize. Crosses $\times$ Environment interaction was also highly significant for all the characters. From the results, it could be concluded that maize genotypes responded significantly for yield and other characters to the environment.

Analysis of variances for combining ability (Table1) indicated that variances for general combining ability (GCA) and specific combining ability (SCA) variance 
were significant for all the characters. However, in the present study variances due to GCA were much higher in magnitude than SCA for all the characters indicating prevalence of additive gene effects for the inheritance of these traits. Malik et al. (2004) in their study also found higher GCA variances than SCA for days to pollen shedding, plant height, ear height, 1000-kernel weight and grain yield. Predominance of additive gene action for various quantitative traits in maize was reported by Muraya et al. (2006), Ahmed et al. (2008) and Amiruzzaman (2010). The current result was in contrast with Abdel-Moneam et al. (2009) who observed GCA/SCA ratio was less than unity for 100-kernels weight and ear yield/plant indicating non additive gene action in controlling the traits. Kadir (2010) in his study showed that the non-additive effects (SCA) were more important than additive effects (GCA) for plant height, ear height, days to pollen shedding, days to silking, 1000-seed weight and grain yield/plant.

Both GCA and SCA effects showed significant interaction with environment for all the traits. This suggested the need of selecting different parental lines for hybrids for different ecological situations. Previous investigations have shown that both GCA and SCA can interact with environments (Rojas and Sprague, 1952; Matzinger et al. 1959; Paroda and Hayes, 1971; Pixley and Bjarnason, 1993; Nass et. al. 2000).

Table1. Analysis of variance for combining ability for yield and yield contributing characters in maize over five environments during rabi 2015-16

\begin{tabular}{l|c|c|c|c|c|c|c}
\hline $\begin{array}{c}\text { Source of } \\
\text { variation }\end{array}$ & df & $\begin{array}{c}\text { Yield } \\
\text { (t/ha) }\end{array}$ & $\begin{array}{c}\text { Days to } \\
\text { pollen } \\
\text { shedding }\end{array}$ & $\begin{array}{c}\text { Days to } \\
\text { silking }\end{array}$ & $\begin{array}{c}\text { Plant } \\
\text { ht.(cm) }\end{array}$ & Ear ht. (cm) & $\begin{array}{c}1000- \\
\text { kernel wt. }\end{array}$ \\
\hline Environment (E) & 4 & $125.17^{* *}$ & $1044.5^{* *}$ & $1301.90^{* *}$ & $5041.59^{* *}$ & $5637.78^{* *}$ & $31949.93^{* *}$ \\
Crosses & 20 & $4.94^{* *}$ & $5.46^{* *}$ & $5.38^{* *}$ & $2719.37^{* *}$ & $1201.84^{* *}$ & $3048.82^{* *}$ \\
Crosses $\times \mathrm{E}$ & 80 & $3.10^{* *}$ & $3.24^{* *}$ & $4.27^{* *}$ & $735.36^{* *}$ & $363.65^{* *}$ & $952.83^{* *}$ \\
$\mathrm{GCA}$ & 6 & $10.51^{* *}$ & $11.87^{* *}$ & $6.52^{* *}$ & $8054.12^{* *}$ & $3636.81^{* *}$ & $2987.07^{* *}$ \\
$\mathrm{SCA}$ & 14 & $2.55^{* *}$ & $2.71^{* *}$ & $4.89^{* *}$ & $433.04^{* *}$ & $158.29^{* *}$ & $3075.29^{* *}$ \\
$\mathrm{GCA} \times \mathrm{E}$ & 12 & $3.20^{* *}$ & $2.56^{* *}$ & $6.34^{* *}$ & $1506.28^{* *}$ & $805.51^{* *}$ & $1186.15^{* *}$ \\
$\mathrm{SCA} \times \mathrm{E}$ & 28 & $3.06^{* *}$ & $3.54^{* *}$ & $3.39^{* *}$ & $404.97^{* *}$ & $174.28^{* *}$ & $852.84^{* *}$ \\
Residuals & 20 & 0.44 & 1.87 & 2.25 & 169.67 & 96.40 & 1106.53 \\
\hline
\end{tabular}

$* * \mathrm{P}=0.01$.

\section{General combining ability (gca) effects}

The GCA effect is shown in Table 2. None of the parents were found to be a good general combiner for all the characters studied. A wide range of variability of GCA effects was observed among the parents. In the present study, for days to pollen shedding, silking, plant and ear height the inbred lines with significant and negative GCA effects were considered as good general combiners. On the other 
hand, for yield and other yield components i.e. 1000-kernel wt., those with significant and positive GCA effects was considered as good general combiners.

For grain yield ( $/$ ha), parents $\mathrm{P}_{3}, \mathrm{P}_{5}$ and $\mathrm{P}_{6}$ showed significant and positive GCA effect. In addition to grain yield, parents $\mathrm{P}_{5}$ were also good general combiner for 1000-kernel weight Sharma et al. (1982) reported that parents with good general combiners for grain yield generally shows good performance for various yield components. Similar views have been given by Malik et al. (2004), Ahmed et al. (2008) and Abdel-Moneam et al. (2009). So, these three parents $\left(\mathrm{P}_{3}, \mathrm{P}_{5}\right.$ and $\left.\mathrm{P}_{7}\right)$ could be used extensively in hybrid breeding program with a view to increasing the yield level.

Parent $\mathrm{P}_{6}$ showed significant and negative GCA for days to pollen shedding. None of the parent showed significant and negative GCA for silking though some parents showed negative values. So, use of this parent might be useful in developing early hybrid variety(s).

Parents $\mathrm{P}_{1}$ and $\mathrm{P}_{2}$ exhibited significant and negative GCA effect for plant height and $\mathrm{P}_{1}, \mathrm{P}_{2}$ and $\mathrm{P}_{3}$ for ear height. Uddin et al. (2006) and Ahmed et al. (2008) also found inbred line(s) as good general combiner for short plant type in their study.

For 1000-kernel weight, $\mathrm{P}_{5}$ was found significant and positive GCA effect. This parent is expected to contribute towards increasing the kernel size. Highly significant and positive gca effects for 1000-kernel weight was observed by Uddin et al. (2006), Alam et al. (2008) and Abdel-Moneam et al. (2009).

Table 2. Estimates of general combining ability effects (GCA) of the parents for yield and yield contributing characters in maize over three environments during rabi 2015-16

\begin{tabular}{l|l|l|l|l|l|l}
\hline \multicolumn{1}{c}{ parents } & $\begin{array}{c}\text { Yield } \\
\text { (t/ha) }\end{array}$ & $\begin{array}{c}\text { Days to } \\
\text { pollen } \\
\text { shedding }\end{array}$ & $\begin{array}{c}\text { Days to } \\
\text { silking }\end{array}$ & $\begin{array}{c}\text { Plant } \\
\text { ht.(cm) }\end{array}$ & Ear ht.(cm) & $\begin{array}{c}1000- \\
\text { kernel wt. }\end{array}$ \\
\hline P1 & -0.03 & 0.16 & -0.10 & $-17.89 * *$ & $-9.67 * *$ & -8.51 \\
P2 & $-0.57 * *$ & -0.08 & -0.38 & $-7.24 * *$ & $-4.22 * *$ & 3.61 \\
P3 & $0.22 * *$ & 0.01 & -0.02 & -2.77 & $-5.76^{* *}$ & 5.19 \\
P4 & $-0.25 * *$ & $0.40^{*}$ & $0.50^{* *}$ & -0.17 & 0.08 & 0.13 \\
P5 & $0.42^{* *}$ & 0.04 & 0.21 & $9.41^{* *}$ & $5.44 * *$ & $10.27 *$ \\
P6 & $0.41^{* *}$ & $-0.42^{* *}$ & -0.23 & $8.63 * *$ & $4.19^{* *}$ & -1.92 \\
P7 & $-0.19 *$ & -0.11 & 0.02 & $10.03 * *$ & $9.94 * *$ & $-12.28^{* *}$ \\
\hline SE (gi) & 0.07 & 0.14 & 0.16 & 1.39 & 1.04 & 3.87 \\
LSD $(5 \%)$ & 0.17 & 0.34 & 0.39 & 3.39 & 2.54 & 9.94 \\
LSD $(1 \%)$ & 0.21 & 0.43 & 0.49 & 4.27 & 3.19 & 11.88 \\
\hline
\end{tabular}

$* \mathrm{P}=0.05, * * \mathrm{P}=0.01$.

$\mathrm{P} 1=\mathrm{CML429}, \mathrm{P} 2=\mathrm{CLG} 1837, \mathrm{P} 3=\mathrm{CML} 285, \mathrm{P} 4=\mathrm{CML451}, \mathrm{P} 5=\mathrm{CML431}, \mathrm{P} 6=$ CML223 and P7= CML551. 


\section{Specific combining ability effects (SCA)}

For grain yield, five crosses $\left(\mathrm{P}_{1} \times \mathrm{P}_{2}, \mathrm{P}_{1} \times \mathrm{P}_{5}, \mathrm{P}_{3} \times \mathrm{P}_{4}, \mathrm{P}_{3} \times \mathrm{P}_{6}, \mathrm{P}_{3} \times \mathrm{P}_{7}\right.$ and $\left.\mathrm{P}_{4} \times \mathrm{P}_{5}\right)$ exhibited significant and positive SCA effects for grain yield. These crosses involved low $\mathrm{x}$ low, low $\mathrm{x}$ high, high $\mathrm{x}$ high and high $\mathrm{x}$ low general combining parents and produced moderate grain yield. Ivy and Hawlader (2000) also reported that good general combining parents do not always show high SCA effects in their hybrid combinations. On the contrary, Paul and Duara (1991) reported that the parents with high GCA always produce hybrids with high estimates of sca. Thus the SCA effects of the crosses were not reflected through the GCA effects of the parents.

Table 3. Specific combining ability effects (SCA) of the crosses for yield and yield contributing characters in maize over three environments during rabi 2015-16

\begin{tabular}{|c|c|c|c|c|c|c|}
\hline Crosses & $\begin{array}{l}\text { Yield } \\
\text { (t/ha) }\end{array}$ & $\begin{array}{l}\text { Days to } \\
\text { pollen } \\
\text { shedding }\end{array}$ & $\begin{array}{l}\text { Days to } \\
\text { silking }\end{array}$ & $\begin{array}{c}\text { Plant } \\
\text { ht. }(\mathrm{cm})\end{array}$ & Ear ht. (cm) & $\begin{array}{c}\text { 1000- } \\
\text { kernel wt. }\end{array}$ \\
\hline $\mathrm{P} 1 \times \mathrm{P} 2$ & $0.71 * *$ & 0.35 & 0.52 & 4.65 & 4.22 & 1.40 \\
\hline $\mathrm{P} 1 \times \mathrm{P} 3$ & $-0.41 * *$ & 0.24 & 0.23 & -1.66 & -1.39 & $-24.61 * *$ \\
\hline $\mathrm{P} 1 \times \mathrm{P} 4$ & $-0.52 * *$ & 0.12 & 0.29 & -0.71 & 0.66 & 5.97 \\
\hline $\mathrm{P} 1 \times \mathrm{P} 5$ & $0.45 * *$ & 0.35 & 0.19 & 2.52 & -3.79 & 12.71 \\
\hline $\mathrm{P} 1 \times \mathrm{P} 6$ & -0.16 & $-0.58 *$ & $-0.75^{*}$ & -2.16 & 0.75 & $19.53 *$ \\
\hline $\mathrm{P} 1 \times \mathrm{P} 7$ & -0.06 & -0.48 & -0.48 & -2.62 & -0.45 & -15.03 \\
\hline $\mathrm{P} 2 \times \mathrm{P} 3$ & $-0.44 * *$ & 0.23 & 0.37 & $-11.87 * *$ & -4.08 & $22.48 * *$ \\
\hline $\mathrm{P} 2 \times \mathrm{P} 4$ & 0.01 & 0.51 & 0.51 & -0.23 & 0.89 & -16.29 \\
\hline $\mathrm{P} 2 \times \mathrm{P} 5$ & $-0.31 *$ & $-0.72 *$ & $-0.72 *$ & 2.4 & 2.65 & 0.52 \\
\hline $\mathrm{P} 2 \times \mathrm{P} 6$ & 0.03 & -0.12 & -0.27 & -1.64 & $-5.31 *$ & -13.26 \\
\hline $\mathrm{P} 2 \times \mathrm{P} 7$ & -0.01 & -0.23 & -0.40 & $6.68 *$ & 1.61 & 5.14 \\
\hline $\mathrm{P} 3 \times \mathrm{P} 4$ & $0.46 * *$ & $-0.99 * *$ & $-1.11 * *$ & 4.14 & 1.13 & -10.10 \\
\hline $\mathrm{P} 3 \times \mathrm{P} 5$ & $-0.35^{*}$ & -0.09 & 0.17 & -2.7 & 0.65 & -13.12 \\
\hline $\mathrm{P} 3 \times \mathrm{P} 6$ & $0.33^{*}$ & 0.16 & 0.29 & $7.28 *$ & -0.78 & 1.31 \\
\hline $\mathrm{P} 3 \times \mathrm{P} 7$ & $0.40 * *$ & 0.25 & 0.03 & 4.87 & $4.48 *$ & $24.07 * *$ \\
\hline $\mathrm{P} 4 \times \mathrm{P} 5$ & $0.42 * *$ & -0.22 & -0.42 & 3.59 & -0.63 & 8.74 \\
\hline $\mathrm{P} 4 \times \mathrm{P} 6$ & -0.12 & 0.24 & 0.36 & -0.87 & 2.22 & 6.91 \\
\hline $\mathrm{P} 4 \times \mathrm{P} 7$ & -0.24 & 0.33 & 0.36 & $-5.91 *$ & -4.2 & 4.76 \\
\hline $\mathrm{P} 5 \times \mathrm{P} 6$ & -0.09 & 0.33 & 0.32 & -2.66 & 2.79 & -2.21 \\
\hline $\mathrm{P} 5 \times \mathrm{P} 7$ & -0.11 & 0.16 & 0.45 & -3.09 & -1.66 & -6.64 \\
\hline $\mathrm{P} 6 \times \mathrm{P} 7$ & 0.02 & -0.11 & 0.04 & 0.07 & 0.31 & -12.28 \\
\hline SE (ij) & 0.14 & 0.28 & 0.31 & 2.74 & 2.06 & 7.84 \\
\hline $\operatorname{LSD}(5 \%)$ & 0.29 & 0.58 & 0.64 & 5.70 & 4.28 & 16.31 \\
\hline $\operatorname{LSD}(1 \%)$ & 0.40 & 0.80 & 0.88 & 7.78 & 5.85 & 22.27 \\
\hline
\end{tabular}

$* \mathrm{P}=0.05, * * \mathrm{P}=0.01$

$\mathrm{P} 1=\mathrm{CML429}, \mathrm{P} 2=\mathrm{CLG} 1837, \mathrm{P} 3=\mathrm{CML} 285, \mathrm{P} 4=\mathrm{CML} 451, \mathrm{P} 5=\mathrm{CML} 431, \mathrm{P} 6=$ CML223 and P7= CML551 
Significant and negative SCA effects for days to pollen shedding and silking are desirable for selection of early maturing hybrids. Three crosses $\left(\mathrm{P}_{1} \times \mathrm{P}_{6}, \mathrm{P}_{2} \times \mathrm{P}_{5}\right.$ and $\mathrm{P}_{3} \times \mathrm{P}_{4}$ ) exhibited significant and negative sca effects for days to pollen shedding and silking. These crosses mostly involved average $\times$ high, average $x$ average, average $\mathrm{x}$ high and average $\mathrm{x}$ low general combining parents. These findings are consistent with the results of Ahmed et al. (2008).

Two crosses $\left(\mathrm{P}_{2} \times \mathrm{P}_{3}\right.$ and $\left.\mathrm{P}_{4} \times \mathrm{P}_{7}\right)$ exhibited significant and negative SCA effects for plant height and one cross $\left(\mathrm{P}_{2} \times \mathrm{P}_{6}\right)$ for ear height which are desirable for exploiting non additive gene. This cross involved high $\mathrm{x}$ average, average $\mathrm{x}$ high and high $\mathrm{x}$ low general combining parents (Table 2 and 3 ).

For 1000-kernel weight, three crosses $\left(\mathrm{P}_{1} \times \mathrm{P}_{6}, \mathrm{P}_{2} \times \mathrm{P}_{3}\right.$ and $\left.\mathrm{P}_{3} \times \mathrm{P}_{7}\right)$ showed significant and positive SCA effects involved low $\times$ low, average $\times$ average and average $\times$ low combining parents and also possessed high mean value.

\section{Heterosis}

The standard/economic heterosis expressed by the $F_{1}$ hybrids over the commercial check variety BHM9 for yield and yield related traits are shown in Table 4. All the traits showed significant heterosis in different crosses.

For grain yield(t/ha) four crosses showed significant and positive heterosis over the standard check variety BHM-9 which was found in the range of -13.04 to $5.25 \%$. Amiruzzaman (2010) and Kadir (2010) in their study found -17.60 to $9.71 \%$ and -15.21 to $27.97 \%$ standard heterosis for kernel yield, respectively.

Significant and negative heterosis was exhibited by all of the crosses both days to pollen shedding and days to silking indicating earliness. Negative heterosis is desirable for plant height which helps to develop short stature plant leading to lodging resistant. Heterosis for different crosses ranged from -18.47 to -1.20 percent and -23.20 to 1.10 percent, respectively, for plant and ear height. Significant and negative heterosis for both these traits was reported by Uddin et al. (2006), Alam et al. (2008) and Amiruzzaman (2010).

Two crosses showed significant and positive heterosis for 1000-kernel weight. Heterosis for different crosses ranged from -8.61 to 9.59 percent.

Results of overall means across five locations from combined analysis are presented in Table 5. Considering overall mean grain yield across locations, three crosses $\mathrm{P}_{1} \times \mathrm{P}_{5}(10.22 \mathrm{t} / \mathrm{ha}), \mathrm{P}_{3} \times \mathrm{P}_{6}(10.36 \mathrm{t} / \mathrm{ha})$ and $\mathrm{P}_{5} \times \mathrm{P}_{6}$ (10.13) out yielded the better check hybrid BHM9 (9.84 t/ha). Days to tasseling and silking, ranged from 85 to 88 days and 87 to 90 days respectively (Table 5). Plant height was found the highest $(238 \mathrm{~cm})$ in check variety BHM9 and the lowest $(194 \mathrm{~cm})$ in $\mathrm{P}_{2}$ $\times \mathrm{P}_{3}$. Ear height was found the highest $(124 \mathrm{~cm})$ in $\mathrm{P}_{6} \times \mathrm{P}_{7}$ and the lowest (93) in $\mathrm{P}_{2} \times \mathrm{P}_{3}$. 1000-kernel weight ranged from 319 to $383 \mathrm{~g}$. 
Table 4. Heterosis of the crosses over BHM9 for different characters in maize over five environments during rabi 2015-16

\begin{tabular}{|c|c|c|c|c|c|c|}
\hline Crosses & $\begin{array}{l}\text { Yield } \\
\text { (t/ha) }\end{array}$ & $\begin{array}{c}\text { Days to } \\
\text { pollen } \\
\text { shedding }\end{array}$ & $\begin{array}{l}\text { Days to } \\
\text { silking }\end{array}$ & $\begin{array}{l}\text { Plant } \\
\text { ht. }(\mathrm{cm})\end{array}$ & $\begin{array}{c}\text { Ear } \\
\text { ht. }(\mathrm{cm})\end{array}$ & $\begin{array}{c}1000- \\
\text { kernel wt. }\end{array}$ \\
\hline $\mathrm{P} 1 \times \mathrm{P} 2$ & $-3.63^{* *}$ & $-1.42 * *$ & $-2.23 * *$ & $-17.69 * *$ & $-17.25 * *$ & -0.36 \\
\hline $\mathrm{P} 1 \times \mathrm{P} 3$ & $-6.98 * *$ & $-1.42 * *$ & $-2.16^{* *}$ & $-18.28 * *$ & $-23.20 * *$ & $-7.35^{* *}$ \\
\hline $\mathrm{P} 1 \times \mathrm{P} 4$ & $-13.04 * *$ & -1.12 & $-1.49 * *$ & $-8.58 * *$ & $-16.64 * *$ & -0.05 \\
\hline $\mathrm{P} 1 \times \mathrm{P} 5$ & $3.88 * *$ & $-1.27 * *$ & $-1.94 * *$ & -3.68 & $-15.89 * *$ & $4.78 *$ \\
\hline $\mathrm{P} 1 \times \mathrm{P} 6$ & $-2.53 * *$ & $-2.86 * *$ & $-3.48 * *$ & -2.61 & $-13.14 * *$ & 3.24 \\
\hline $\mathrm{P} 1 \times \mathrm{P} 7$ & $-7.76 * *$ & $-2.41 * *$ & $-2.89 * *$ & -1.20 & $-9.38 * *$ & $-8.61 * *$ \\
\hline $\mathrm{P} 2 \times \mathrm{P} 3$ & $-12.68 * *$ & $-1.73 * *$ & $-2.30 * *$ & $-18.47 * *$ & $-20.91 * *$ & $9.59 * *$ \\
\hline $\mathrm{P} 2 \times \mathrm{P} 4$ & $-13.04 * *$ & $-0.97 * *$ & $-1.57 * *$ & $-12.30 * *$ & $-11.92 * *$ & -2.95 \\
\hline $\mathrm{P} 2 \times \mathrm{P} 5$ & $-9.33 * *$ & $-2.79 * *$ & $-3.26 * *$ & $-7.45^{*}$ & $-6.01 * *$ & 4.76 \\
\hline $\mathrm{P} 2 \times \mathrm{P} 6$ & $-5.91 * *$ & $-2.64 * *$ & $-3.26 * *$ & $-5.89^{*}$ & $-13.65 * *$ & -2.68 \\
\hline $\mathrm{P} 2 \times \mathrm{P} 7$ & $-12.54 * *$ & $-2.41 * *$ & $-3.12 * *$ & -2.20 & -3.13 & 0.63 \\
\hline $\mathrm{P} 3 \times \mathrm{P} 4$ & $-0.28 * *$ & $-2.56 * *$ & $-2.97 * *$ & $-16.98 * *$ & $-12.99 * *$ & -0.73 \\
\hline $\mathrm{P} 3 \times \mathrm{P} 5$ & -1.61 & $-1.73 * *$ & $-1.86 * *$ & $-7.16^{*}$ & $-8.95 * *$ & 1.31 \\
\hline $\mathrm{P} 3 \times \mathrm{P} 6$ & $5.25 * *$ & $-2.18 * *$ & $-2.23 * *$ & -3.56 & $-11.18 * *$ & 1.95 \\
\hline $\mathrm{P} 3 \times \mathrm{P} 7$ & $-0.29 * *$ & $-1.73 * *$ & $-2.23 * *$ & $-7.42^{*}$ & -2.03 & $6.49 *$ \\
\hline $\mathrm{P} 4 \times \mathrm{P} 5$ & $1.37 * *$ & $-1.65 * *$ & $-1.94 * *$ & $-11.58 * *$ & $-5.16 * *$ & 6.12 \\
\hline $\mathrm{P} 4 \times \mathrm{P} 6$ & $-4.39 * *$ & $-1.65 * *$ & $-1.57 * *$ & $-9.19 * *$ & -3.82 & 2.10 \\
\hline $\mathrm{P} 4 \times \mathrm{P} 7$ & $-11.78 * *$ & $-1.19 * *$ & $-1.27 * *$ & -3.98 & $-4.46^{*}$ & -0.47 \\
\hline $\mathrm{P} 5 \times \mathrm{P} 6$ & $2.89 * *$ & $-1.95 * *$ & $-1.94 * *$ & $-13.88 * *$ & 1.10 & 2.39 \\
\hline $\mathrm{P} 5 \times \mathrm{P} 7$ & $-3.57 * *$ & $-1.80 * *$ & $-1.49 * *$ & -5.09 & 2.17 & -0.84 \\
\hline $\mathrm{P} 6 \times \mathrm{P} 7$ & $-2.27 * *$ & $-2.56 * *$ & $-2.45^{* *}$ & $-13.49 * *$ & 2.78 & -5.94 \\
\hline Minimum & -13.04 & -2.86 & -3.48 & -18.47 & -23.2 & -8.61 \\
\hline Maximum & 5.25 & -1.12 & -1.27 & -1.20 & 1.10 & 9.59 \\
\hline $\operatorname{LSD}(5 \%)$ & 0.29 & 0.25 & 0.30 & 5.73 & 3.66 & 9.59 \\
\hline $\operatorname{LSD}(1 \%)$ & 0.40 & 0.34 & 0.40 & 7.77 & 4.97 & 8.64 \\
\hline
\end{tabular}

$* \mathrm{P}=0.05, * * \mathrm{P}=0.01$.

$\mathrm{P} 1=$ CML429, $\mathrm{P} 2=\mathrm{CLG} 1837, \mathrm{P} 3=\mathrm{CML} 285, \mathrm{P} 4=\mathrm{CML} 451, \mathrm{P} 5=\mathrm{CML} 431, \mathrm{P} 6=$ CML223 and P7= CML551. 
Table 5. Mean performance of the crosses for yield and yield contributing characters in maize over five environments during rabi 2015-16

\begin{tabular}{|c|c|c|c|c|c|c|}
\hline Crosses & $\begin{array}{l}\text { Yield } \\
(\mathrm{t} / \mathrm{ha})\end{array}$ & $\begin{array}{c}\text { Days to } \\
\text { pollen } \\
\text { shedding }\end{array}$ & $\begin{array}{l}\text { Days to } \\
\text { silking }\end{array}$ & $\begin{array}{c}\text { Plant } \\
\text { ht. }(\mathrm{cm})\end{array}$ & Ear ht. $(\mathrm{cm})$ & $\begin{array}{c}1000- \\
\text { kernel wt. } \\
(\mathrm{g})\end{array}$ \\
\hline $\mathrm{P} 1 \times \mathrm{P} 2$ & 9.48 & 87 & 88 & 196 & 100 & 348 \\
\hline $\mathrm{P} 1 \times \mathrm{P} 3$ & 9.15 & 87 & 88 & 194 & 93 & 324 \\
\hline $\mathrm{P} 1 \times \mathrm{P} 4$ & 8.56 & 87 & 89 & 217 & 100 & 349 \\
\hline $\mathrm{P} 1 \times \mathrm{P} 5$ & 10.22 & 87 & 89 & 229 & 101 & 366 \\
\hline $\mathrm{P} 1 \times \mathrm{P} 6$ & 9.59 & 85 & 87 & 232 & 105 & 361 \\
\hline $\mathrm{P} 1 \times \mathrm{P} 7$ & 9.08 & 86 & 88 & 235 & 109 & 319 \\
\hline $\mathrm{P} 2 \times \mathrm{P} 3$ & 8.59 & 86 & 88 & 194 & 95 & 383 \\
\hline $\mathrm{P} 2 \times \mathrm{P} 4$ & 8.56 & 87 & 89 & 209 & 106 & 339 \\
\hline $\mathrm{P} 2 \times \mathrm{P} 5$ & 8.92 & 85 & 87 & 220 & 113 & 366 \\
\hline $\mathrm{P} 2 \times \mathrm{P} 6$ & 9.26 & 86 & 87 & 224 & 104 & 340 \\
\hline $\mathrm{P} 2 \times \mathrm{P} 7$ & 8.61 & 86 & 88 & 233 & 117 & 352 \\
\hline $\mathrm{P} 3 \times \mathrm{P} 4$ & 9.81 & 86 & 88 & 198 & 105 & 347 \\
\hline $\mathrm{P} 3 \times \mathrm{P} 5$ & 9.68 & 86 & 89 & 221 & 110 & 354 \\
\hline $\mathrm{P} 3 \times \mathrm{P} 6$ & 10.36 & 86 & 88 & 229 & 107 & 356 \\
\hline $\mathrm{P} 3 \times \mathrm{P} 7$ & 9.81 & 86 & 88 & 220 & 118 & 372 \\
\hline $\mathrm{P} 4 \times \mathrm{P} 5$ & 9.98 & 86 & 89 & 210 & 114 & 371 \\
\hline $\mathrm{P} 4 \times \mathrm{P} 6$ & 9.41 & 86 & 89 & 216 & 116 & 357 \\
\hline $\mathrm{P} 4 \times \mathrm{P} 7$ & 8.68 & 87 & 89 & 228 & 115 & 348 \\
\hline $\mathrm{P} 5 \times \mathrm{P} 6$ & 10.13 & 86 & 89 & 205 & 122 & 358 \\
\hline $\mathrm{P} 5 \times \mathrm{P} 7$ & 9.49 & 86 & 89 & 226 & 123 & 347 \\
\hline $\mathrm{P} 6 \times \mathrm{P} 7$ & 9.62 & 86 & 88 & 206 & 124 & 329 \\
\hline BHM9 & 9.84 & 88 & 90 & 238 & 120 & 350 \\
\hline 981 & 11.47 & 86 & 89 & 228 & 109 & 351 \\
\hline NK40 & 9.81 & 86 & 89 & 211 & 107 & 340 \\
\hline F-test & $* *$ & $* *$ & $* *$ & $* *$ & $* *$ & $* *$ \\
\hline Minimum & 8.56 & 85 & 87 & 194 & 93 & 319 \\
\hline Maximum & 11.47 & 88 & 90 & 238 & 124 & 383 \\
\hline
\end{tabular}

$* \mathrm{P}=0.05, * * \mathrm{P}=0.01$.

$\mathrm{P} 1=\mathrm{CML} 429, \mathrm{P} 2=\mathrm{CLG} 1837, \mathrm{P} 3=\mathrm{CML} 285, \mathrm{P} 4=\mathrm{CML} 451, \mathrm{P} 5=\mathrm{CML431}, \mathrm{P} 6=$ CML223 and P7= CML551. 


\section{Conclusion}

Both GCA and SCA effects showed significant interaction with environment for all of the traits. This suggested the need of selecting different parental lines for hybrids for different ecological situations. Parents with good positive GCA for yield $\left(\mathrm{P}_{5}\right.$ and $\left.\mathrm{P}_{6}\right)$ and 1000- kernel wt. $\left(\mathrm{P}_{5}\right)$; and negative GCA for days to pollen shedding and silking $\left(\mathrm{P}_{6}\right.$ and $\left.\mathrm{P}_{7}\right)$, plant height and ear height $\left(\mathrm{P}_{1}, \mathrm{P}_{2}\right.$ and $\left.\mathrm{P}_{3}\right)$ might be extensively used in hybridization program as a donor. The better performing six crosses $\left(\mathrm{P}_{1} \times \mathrm{P}_{2}, \mathrm{P}_{1} \times \mathrm{P}_{5}, \mathrm{P}_{3} \times \mathrm{P}_{4}, \mathrm{P}_{3} \times \mathrm{P}_{6}, \mathrm{P}_{3} \times \mathrm{P}_{7}\right.$ and $\left.\mathrm{P}_{4} \times \mathrm{P}_{5}\right)$ could be utilized for developing high yielding hybrid varieties as well as for exploiting hybrid vigour. These crosses also need to be evaluated further in wider agro-climatic conditions. Additionally, it could be concluded that all these selected crosses could be treated as well buffered hybrids.

\section{References}

Abdel-Moneam, M.A., A.N. Attia, M.I. El-Emery and E.A. Fayed. 2009. Combining ability and heterosis for some agronomic traits in crosses of maize. Pakistan J. Biol. Sci. 12(5): 433-438.

Ahmad. S.Q., S. Khan, M. Ghaffar and P. Ahmad. 2011. Genetic diversity analysis for yieldand other parameters in maize (Zea mays L.) Genotype. Asian J. Agril. Sci. 3(5): 385-388.

Ahmed, S., F. Khatun, M.S. Uddin, B.R. Banik and N. A. Ivy. 2008. Combining ability and heterosis in maize (Zea mays L.). Bangladesh J. Pl. Breed. Genet. 21(2): 27-32.

Alam, A.K.M.M., S. Ahmed, M. Begum and M.K. Sultan. 2008. Heterosis and combining ability for grain yield and its contributing characters in maize. Bangladesh J. Agril. Res. 33(3): 375-379.

Amiruzzaman, M. 2010. Exploitation of hybrid vigour from normal and quality protein maize crosses. Ph.D Dissertation, Dept. Genetics and Plant Breeding, Bangladesh Agricultural University, Mymensingh, 200 P.

Bidan, B. 2016. Agriculture Market information system in Bangladesh.Thimpu, Bhutan. Pp. 15-19.

Griffings, B. 1956. Concept of general and specific combining in relation of diallel crossing systems. Australian J. Bio. Sci. 9: 463-93.

Ivy, N.A. and M.S. Howlader. 2000. Combining ability in maize. Bangladesh J. Agril. Res., 25: 385-392.

Kadir, M.M. 2010. Development of quality protein maize hybrids and their adaptation in Bangladesh. PhD Dissertation, Dept. Genetics \& Plant Breeding, Bangladesh Agricultural University, Mymensingh.

Malik S.I., H.N. Malik, N.M. Minhas and M. Munir. 2004. General and specific combining ability studies in maize diallel crosses. Intl. J. of Agric. and Biology. 6(5): 856-859.

Matzinger, D.F., G.F. Sprague and C.C. Cockerham. 1959. Diallel cross of maize in experiments repeated over locations and years. J. Agron. 51: 346-350. 
Muraya, M.M., C.M. Ndirangu and E.O. Omolo. 2006. Heterosis and combining ability in diallel crosses involving maize (Zea mays) S1 lines. Australian Journal of Experimental Agriculture. 46: 387-394.

Nass, L.L., R. Lima, R. Vencovsky and P.B. Gallo. 2000. Combining ability of maize inbred lines evaluated in three environments in Brazil. Sci. Agric. 57(1).

Paroda, R.S. and J.D. Hayes. 1971. An investigation of genotype-environment interactions for rate of ear emergence in spring barley. Heredity. 26:157-175.

Paul, S.K. and R.K. Duara. 1991. Combining ability studies in maize (Zea mays L.). Intl. J. Tropic. Agric. 9: 250-254.

Pixley, K.V. and M.S. Bjarnason. 1993. Combining ability for yield and protein quality among modified-endosperm opaque-2 tropical maize inbreds. Crop Sci. 33: 12291234.

Rojas, B.A. and G.F. Sprague. 1952. A comparison of variance components in corn yield trials: III. General and specific combining ability and their interaction with locations and years. J. Agron. 44: 462-466.

Sharma, S. R., A. S. Khera, B. S. Dhillion and V. V. Malhotra. 1982. Evaluation of $\mathrm{S}_{1}$ lines of maize crossed in a diallel system. Crop Improv. 9(1): 42-47.

Uddin, S.M., F. Khatun, S. Ahmed, M.R. Ali and S.A. Begum. 2006. Heterosis and combining ability in corn (Zea mays L.). Bangladesh J. Bot. 35: 109-116. 